Supporting Information

\title{
Photosystem I Enhances the Efficiency of a Natural, Gel-Based Dye-Sensitized Solar Cell
}

\author{
Joshua M. Passantino,${ }^{\dagger}$ Kody D. Wolfe, ${ }^{\dagger}$ Keiann T. Simon,${ }^{\dagger}$ David E. Cliffel, ${ }^{\S}$ G. Kane \\ Jennings* ${ }^{*} \dagger$ \\ 'Department of Chemical and Biomolecular Engineering, Vanderbilt University, Nashville, Tennessee 37235, \\ United States \\ \#nterdisciplinary Materials Science Program, Vanderbilt University, Nashville, Tennessee 37235, United States

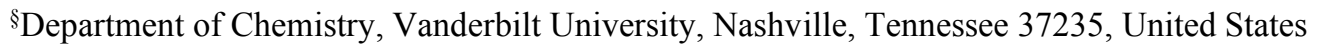 \\ *Corresponding author: kane.g.jennings@vanderbilt.edu
}

\section{List of Figures:}

Fig. S1: Comparison of mediator performance on a copper working electrode with a PSI film. $\mathrm{Ag} / \mathrm{AgCl}$ was used as a reference electrode along with a Pt counter electrode and a $100 \mathrm{mM} \mathrm{KCl(aq)} \mathrm{supporting}$ electrolyte.

Fig. S2: Energy diagram of the AscH/DCPIP reaction with PSI on a copper electrode.

Fig. S3: Profilometric thickness of a characteristic vacuum-assisted, drop-cast PSI multilayer film.

Fig. S4: PM-IRRAS Spectra of a PSI multilayer film deposited on copper.

Fig. S5: Dependence of $\mathrm{pH}$ on peak currents in cyclic voltammetry (scan rate $=100 \mathrm{mV} / \mathrm{s}$ ) for $1 \mathrm{mM}$ DCPIP in $100 \mathrm{mM}$ phosphate buffer. The electrodes consisted of Au (working), Pt (counter), and $\mathrm{Ag} / \mathrm{AgCl}$ (reference).

Fig. S6: Forward and reverse scan j-V curves at $1 \mathrm{~V} / \mathrm{s}$ for devices at neutral $\mathrm{pH}$, with and without PSI, and a mediator concentration of $20 \mathrm{mM}$ AscH to $1 \mathrm{mM}$ DCPIP.

Fig. S7: Reverse scan j-V curves at $10 \mathrm{~V} / \mathrm{s}$ for devices at neutral $\mathrm{pH}$, with and without PSI, and a mediator concentration of $20 \mathrm{mM}$ AscH to $1 \mathrm{mM}$ DCPIP.

Fig. S8: Electrochemical impedance spectra of copper electrodes with and without PSI films in $0.5 \mathrm{wt} \%$ agarose gel.

\section{Number of Pages: 8}




\section{Mediator Comparisons with PSI on Copper}

To optimize the composition of the gel-based devices, various mediator options were explored. The mediators were compared by two-electrode photochronoamperometry (PCA) tests to determine photocurrent response with a PSI film placed on a copper cathode in a $100 \mathrm{mM} \mathrm{KCl}$ supporting electrolyte. Methyl viologen (MV) is a good acceptor of electrons from the $\mathrm{F}_{\mathrm{B}}$ site of PSI, and in this setup MV was not expected to perform well because MV accepts electrons from PSI but is not readily oxidized at the electrode. Ferri- and ferrocyanide $(0.5 \mathrm{mM}$ and $0.5 \mathrm{mM}$, respectively) and AscH/DCPIP ( $20 \mathrm{mM} / 1 \mathrm{mM})$ were both investigated because ferrocyanide is known to be a slow donator to $\mathrm{P}_{700}$ while reduced DCPIP is a fast donor, which should lead to significant performance differences with PSI at a copper cathode. AscH/DCPIP is expected to perform better than ferri-/ferrocyanide because an excess of oxidized species in the form of DCPIP would be produced by PSI near the cathode. The PCA results (Figure S1) showed that AscH/DCPIP was the best mediator choice in this setup.

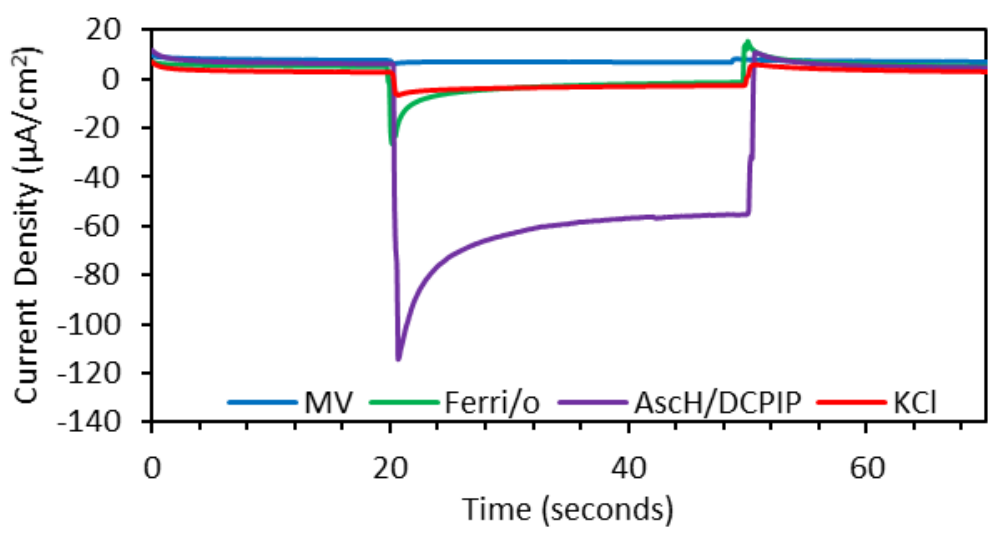

Figure S1. Comparison of mediators on a copper electrode containing $4 \square \mathrm{m}$-thick PSI film. $\mathrm{Ag} / \mathrm{AgCl}$ was used as a reference electrode and $\mathrm{Pt}$ as a counter electrode with a $100 \mathrm{mM} \mathrm{KCl}$ supporting electrolyte.

\section{AscH/DCPIP Reaction Scheme}

The reaction between AscH and DCPIP is a catalytic electron transfer/homogenous chemical reaction couple (EC') that catalytically generates $\mathrm{DCPIPH}_{2}$ by the following mechanism at neutral $\mathrm{pH}:{ }^{1}$

$$
\begin{gathered}
D C P I P+A s c H^{-} \rightleftarrows D C P I P H^{\bullet}+A s c^{\cdot-} \\
D C P I P H^{\bullet}+A_{S C H} \rightleftarrows D C P I P H_{2}+A s c^{\cdot-}
\end{gathered}
$$


Two ascorbate radicals $\left(\mathrm{AsC}^{--}\right)$then react with surrounding protons to regenerate $\mathrm{AscH}^{-}$and dehydroascorbic acid (DHA) as shown:

$$
2 \mathrm{Asc}^{\cdot-}+\mathrm{H}^{+} \rightleftarrows A s C \mathrm{H}^{-}+\mathrm{DHA}
$$

At low $\mathrm{pH}$, the prolonged existence of the ascorbate radical becomes unfavorable and the reaction becomes:

$$
\begin{gathered}
D C P I P+A s c H^{-} \rightleftarrows D C P I P H^{\bullet}+A s c^{\cdot-} \\
D C P I P H^{\bullet}+A s c^{\cdot-}+H^{+} \rightleftarrows D C P I P H_{2}+D H A
\end{gathered}
$$

As shown in the energy diagram (Figure S2), PSI is able to generate a redox cycle with the $\mathrm{AscH} / \mathrm{DCPIP}$ mediator because DCPIP, DHA , and Asc ${ }^{*}$ are capable of accepting electrons from $\mathrm{F}_{\mathrm{B}}$ and reacting with surrounding protons to regenerate their oxidized forms. ${ }^{2,3}$ DCPIP has been shown to be an electron acceptor that can compete with methyl viologen, a rapid and well known $F_{B}$ electron acceptor, suggesting that its reaction with PSI is faster than the reactions of DHA or $\mathrm{Asc}^{\cdot-3} \mathrm{DCPIPH}_{2}$ is a rapid electron donor to $\mathrm{P}_{700}$ and reacts faster than DCPIP with $\mathrm{F}_{\mathrm{B}}$, which leads to an excess of oxidized species around PSI.

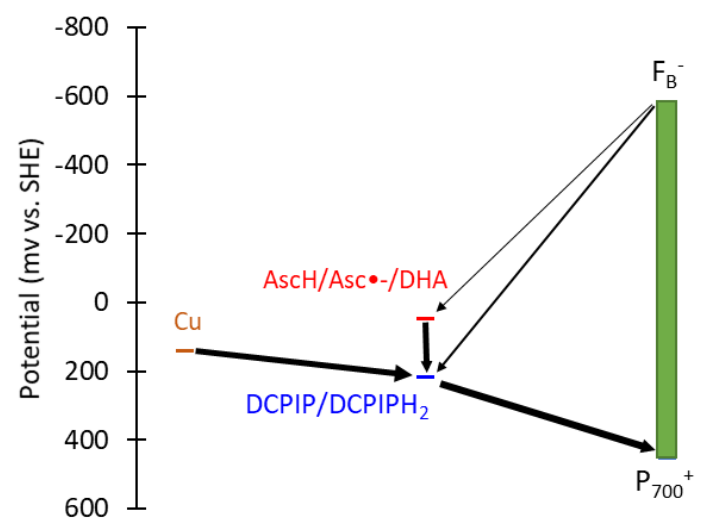

Figure S2. Energy diagram showing the redox cycle of the AscH/DCPIP mediator in the presence of PSI on a copper cathode at $\mathrm{pH}$ 7. Black lines show the electron flow direction. The potential of the copper electrode was found to be $140 \mathrm{mV}$ vs. SHE by open-circuit potential measurements. 


\section{PSI Film Characterization}

PSI was deposited on copper via vacuum-assisted drop casting from an aqueous solution containing $4 \square$ M PSI. After deposition, the thickness of the films was measured via profilometry (Figure S3). Because the PSI multilayer acts as a redox film in this device, a thicker film can provide more reaction sites. The average thickness of the films was $4 \mu \mathrm{m}$.

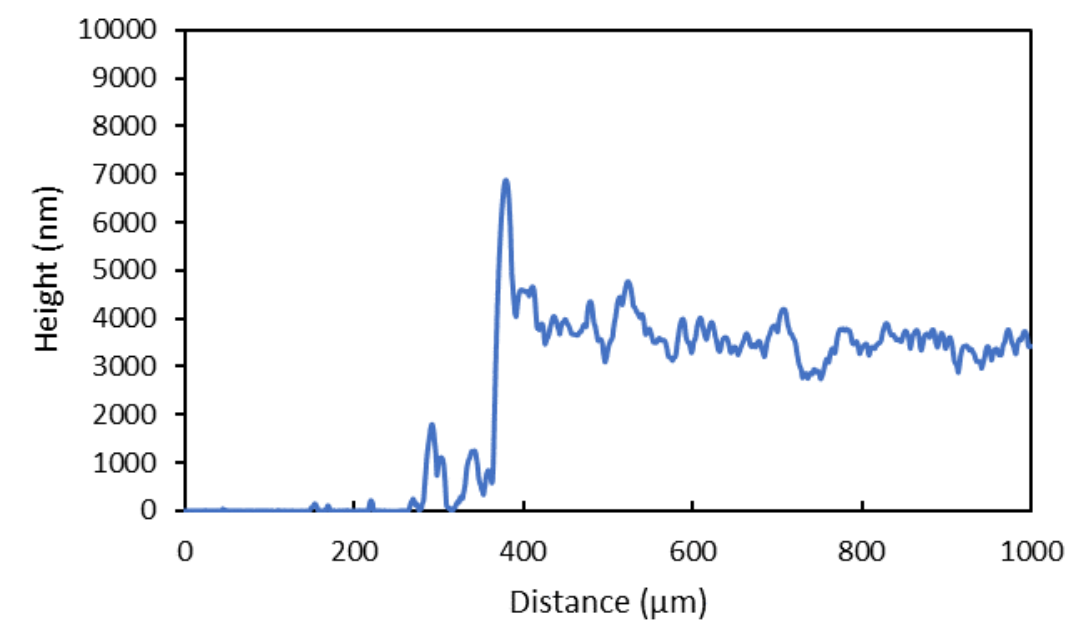

Figure S3. Profilometric thickness of a characteristic vacuum-assisted, drop-cast PSI multilayer film.

To confirm successful deposition of PSI without damaging the protein, PM-IRRAS was conducted on PSI films deposited on copper using an FTIR spectrometer at a resolution of $1 \mathrm{~cm}^{-1}$. The resulting spectra (Figure S4) show strong absorption at peaks at 1662 and $1545 \mathrm{~cm}^{-1}$ representing the Amide I and Amide II peaks characteristic of PSI with low absorption in other regions, suggesting a clean and successful deposition.

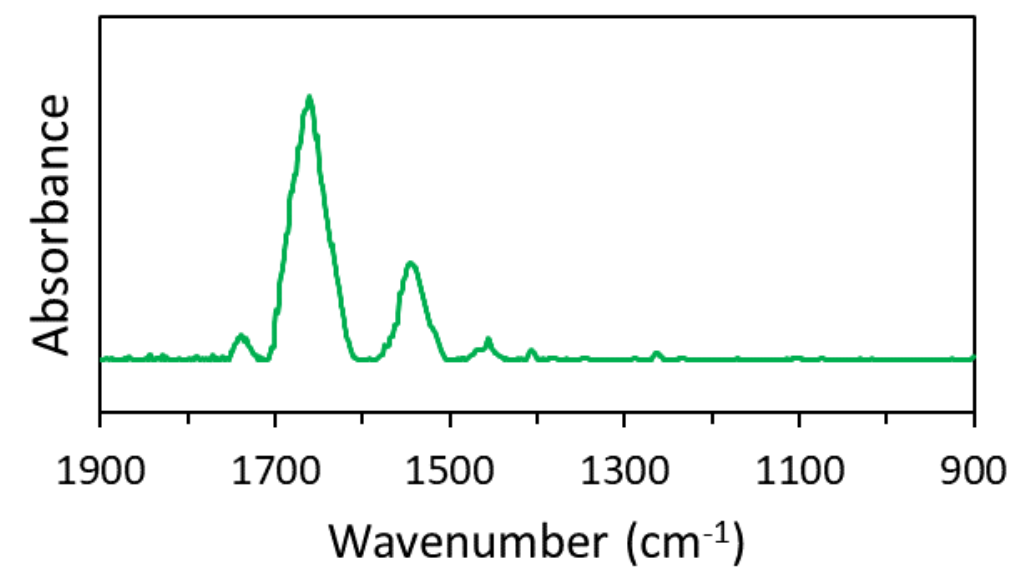

Figure S4. PM-IRRAS FTIR spectra of a PSI multilayer film deposited on copper. 


\section{DCPIP pH Dependence}

DCPIP is known to have different $\mathrm{pH}$-dependent forms that can affect its electrochemical reactivity. Cyclic voltammetry was used to measure the peak currents and confirm reversibility at various pH values ranging from 1.91 to 8.40 (Figure S5). A Au working electrode, Pt counter electrode, and $\mathrm{Ag} / \mathrm{AgCl}$ reference electrode were used in a $100 \mathrm{mM}$ phosphate buffer with $1 \mathrm{mM}$ DCPIP to measure the CVs. The peak currents are expected to be representative of the resulting electrochemical performance of the mediator within the device. DCPIP has two pKa's at 0.52 and 5.6 with the three forms $\mathrm{DH}_{2}{ }^{+}, \mathrm{DH}, \mathrm{D}^{-}$ in order of increasing $\mathrm{pH}$. Based on the $\mathrm{CV}$ results, a $\mathrm{pH}$ of 7.48 yielded the highest peak current, while the lowest peaks were found at 2.77, 3.91, and 5.10. Similarly, Petrova et al. found the reaction with PSI and DCPIP to peak at a $\mathrm{pH}$ of 7.8 when looking at reaction kinetics in solution. ${ }^{3}$ At $\mathrm{pH} 7.48$, DCPIP- $^{-}$is the dominant form, suggesting $\mathrm{D}^{-}$would perform better electrochemically in the devices of this investigation. At $\mathrm{pH} 1.91$, there was an increase in peak current compared to the lowest peaks, likely caused by the formation of $\mathrm{DH}_{2}{ }^{+}$along with $\mathrm{DH}$, but the reversibility was adversely affected based on the large peak separation between anodic and cathodic scans, and we would not want to subject PSI to such acidic conditions.

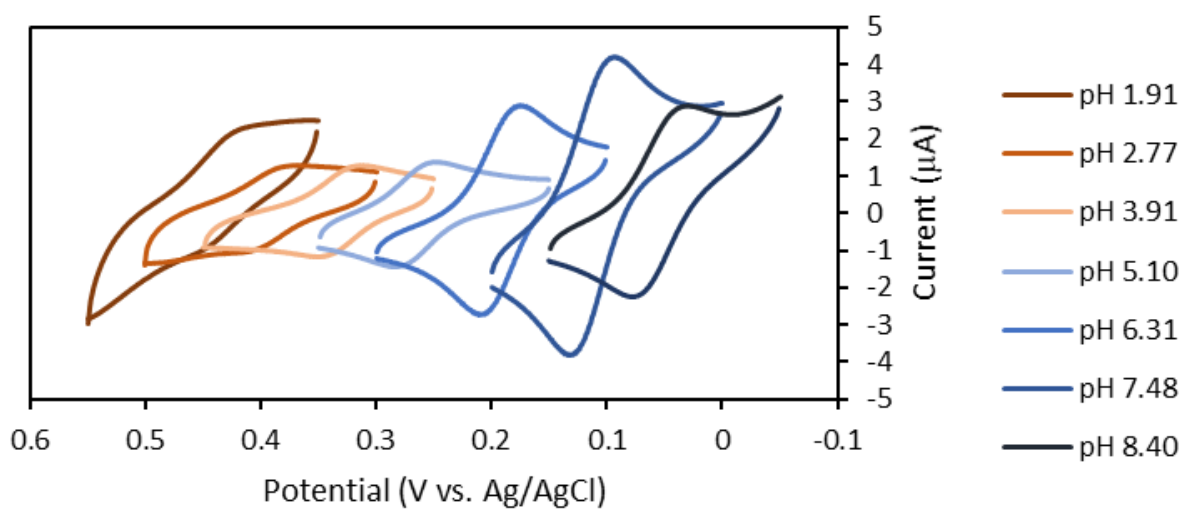

Figure S5. CV pH dependence on peak currents for $1 \mathrm{mM}$ DCPIP in $100 \mathrm{mM}$ phosphate buffer. $\mathrm{Au}$ working electrode, $\mathrm{Pt}$ counter electrode, and $\mathrm{Ag} / \mathrm{AgCl}$ reference. 


\section{j-V Curves}

Forward and reverse scan $\mathrm{j}-\mathrm{V}$ curves were measured to determine the power output of the completed devices (Figure S6 \& Figure S7). The reverse scan in which potential is decreased to approach maximum current provides much improved performance as compared to the forward scan and represents an instantaneous power output. The poor performance of the forward scan is likely due to a depletion of charge carriers by starting the scan at the highest current. Hysteresis in dye-sensitized solar cells is common because of charge carrier trapping in the bulk $\mathrm{TiO}_{2}$. Additionally, the diffusional nature of the cell likely also adds to the hysteresis. For the forward and reverse scans, the important photovoltaic parameters $\left(\mathrm{FF}, \mathrm{J}_{\mathrm{SC}}, \mathrm{V}_{\mathrm{OC}}\right.$, and $\eta$ ) were measured for each scan and are shown in Table 1 in the main text. To determine a more accurate measure of steady-state device performance, the forward and the reverse scans with and without PSI were averaged and the values are also shown in Table 1. A maximum powerpoint voltage was found from the averaged power curves, and steady-state power generation was measured at the maximum power-point voltage to get an accurate power output.

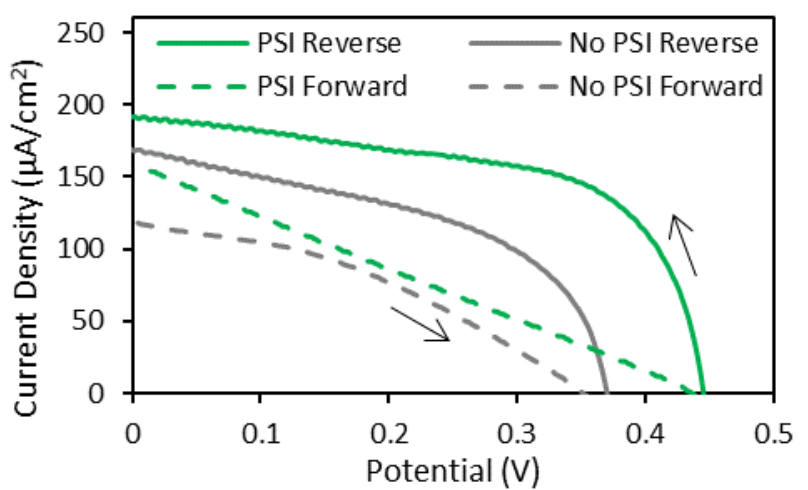

Figure S6. Forward and reverse scan $\mathrm{j}-\mathrm{V}$ curves for devices at neutral $\mathrm{pH}$, with and without PSI, and a mediator concentration of $20 \mathrm{mM}$ AscH to $1 \mathrm{mM}$ DCPIP. Scan rate was $1 \mathrm{~V} / \mathrm{s}$. Samples were illuminated for $30 \mathrm{~s}$ at $80 \mathrm{~mW} / \mathrm{cm}^{2}$ before scans were taken while still under illumination. 


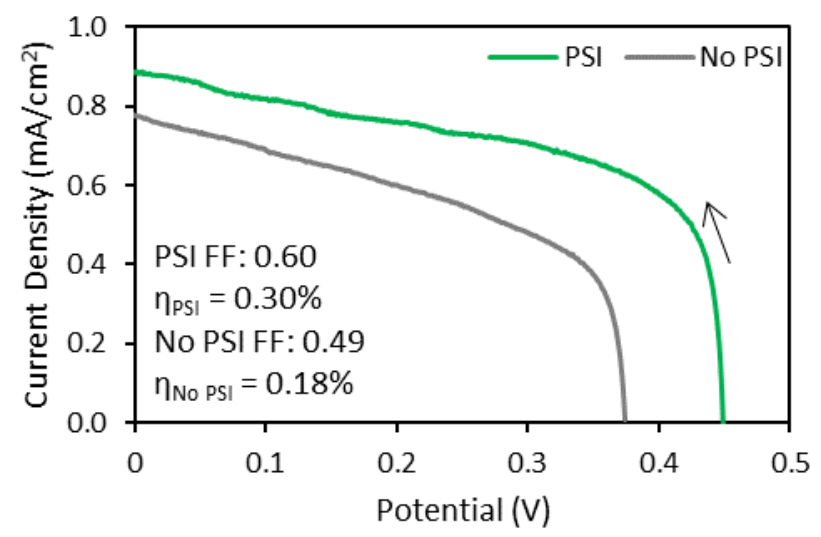

Figure S7. Reverse scan $\mathrm{j}-\mathrm{V}$ curves for devices at neutral $\mathrm{pH}$, with and without PSI, and a mediator concentration of $20 \mathrm{mM}$ AscH to $1 \mathrm{mM}$ DCPIP. Scan rate was $10 \mathrm{~V} / \mathrm{s}$. Samples were illuminated for $30 \mathrm{~s}$ at $80 \mathrm{~mW} / \mathrm{cm}^{2}$ before scans were taken while still under illumination.

\section{PSI Film Effect on Transport Properties}

To determine if the presence of PSI multilayer film affected the charge transport to the cathode, EIS was performed on copper electrodes with and without PSI in a $0.5 \mathrm{wt} \%$ agarose gel (Figure S8). The EIS results show similar charge transfer resistance (low frequencies), capacitance (medium frequencies), and solution resistance (high frequencies) with and without PSI.

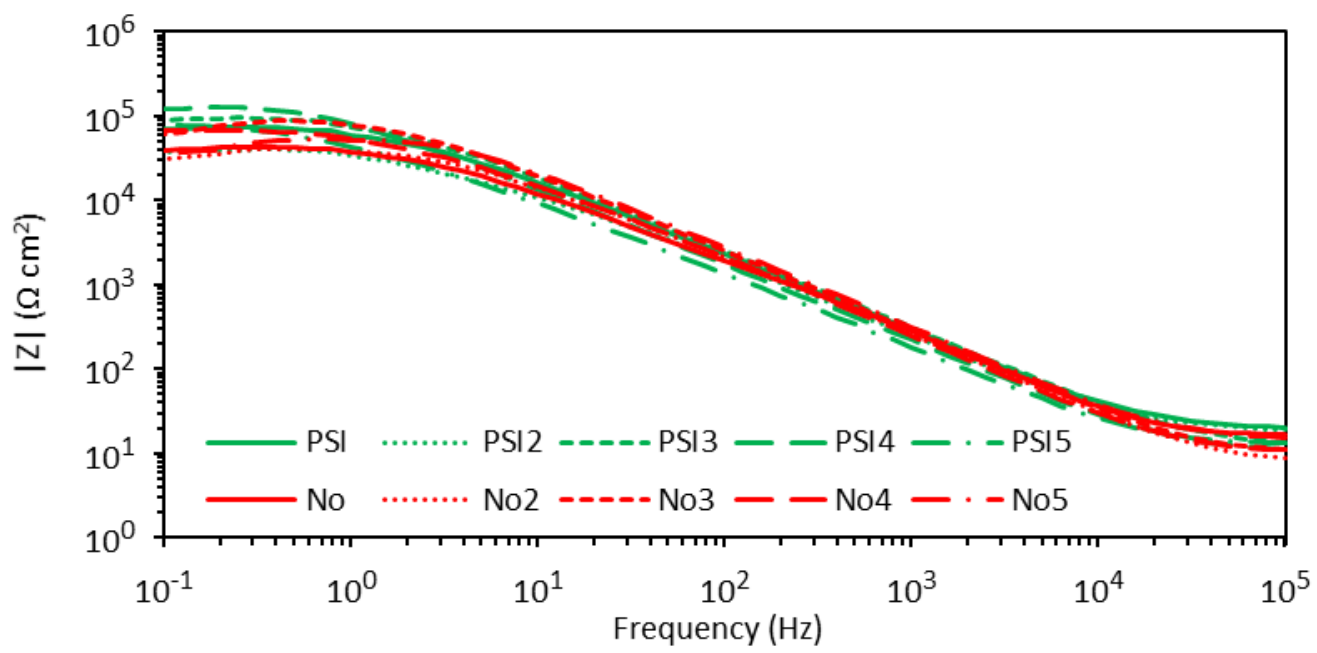

Figure S8. Electrochemical impedance spectra of copper electrodes with and without PSI films in 0.5 wt $\%$ agarose gel. The electrolyte solution for each sample consists of $20 \mathrm{mM} \mathrm{AscH,} 1 \mathrm{mM}$ DCPIP, and $100 \mathrm{mM} \mathrm{KCl}$. 


\section{References}

(1) Marchanka, A.; Van Gastel, M. Reversed Freeze Quench Method near the Solvent Phase Transition. J. Phys. Chem. A 2012, 116 (15), 3899-3906. https://doi.org/10.1021/jp300555x.

(2) Trubitsin, B. V.; Mamedov, M. D.; Semenov, A. Y.; Tikhonov, A. N. Interaction of Ascorbate with Photosystem I. Photosynth. Res. 2014, 122 (2), 215-231. https://doi.org/10.1007/s11120-0140023-7.

(3) Petrova, A.; Mamedov, M.; Ivanov, B.; Semenov, A.; Kozuleva, M. Effect of Artificial Redox Mediators on the Photoinduced Oxygen Reduction by Photosystem I Complexes. Photosynth. Res. 2018, 137 (3), 421-429. https://doi.org/10.1007/s11120-018-0514-z. 